\title{
Das autonome Subjekt: \\ Ermöglichungsbedingung oder Produkt sozialer Praktiken? - Eine Einleitung
}

\author{
Martin Weichold, Jan-Christoph Marschelke, \\ Peter Wiersbinski und Falk Hamann
}

Im Rahmen des vorliegenden Themenschwerpunkts wird das autonome Subjekt, d.h. die Subjektvorstellung, die Recht und Moral zugrunde liegt, aus praxeologischer Perspektive betrachtet. Eine solche Untersuchung ermöglicht zu erkennen, inwiefern das Subjektverständnis des Rechts und der Moral aus sozialtheoretischer Perspektive möglicherweise zeit- und kulturspezifisch ist, inwiefern es aus kognitionswissenschaftlicher Perspektive möglicherweise empirisch zweifelhaft und inwiefern es aus politischer Perspektive eventuell reformbedürftig ist.

Aber worin genau besteht überhaupt die Subjektvorstellung, die Recht und Moral zugrunde liegt? Zugegebenermaßen ist es eine grobe Vereinfachung, von der einen Subjektvorstellung des Rechts und der Moral zu sprechen. Die menschlichen Subjektvorstellungen haben sich in den letzten Jahrtausenden und selbst in den letzten Jahrzehnten immer wieder verändert. ${ }^{1}$ So kann auch das Subjektverständnis im über lange Zeit hinweg entstandenen geltenden Recht im Wesentlichen nur eine Collage unterschiedlicher Subjektverständnisse sein. Dennoch, so schlagen wir vor, lässt sich ein klarer Kern identifizieren, der sich sowohl im Recht als auch in der Moral findet (ganz unabhängig davon, wie man das Verhältnis von Recht und Moral genau bestimmt): Recht und Moral sehen den Menschen als autonomes Subjekt an, das im Rahmen vernünftiger Überlegungen seinen eigenen freien Willen ausbilden und diesen kontrolliert in eine Handlung umsetzen kann. Dabei wüssten die autonomen Subjekte normalerweise, was sie warum tun, könnten sich stets für das Recht und gegen das Unrecht entscheiden und seien eben deshalb für ihre Taten verantwortlich und ihrer Verbrechen schuldig. Das Subjekt sei dabei eine Person, der Menschenwürde zukomme, und eine Trägerin von Rechten und Pflichten. Zugleich impliziert das rechtliche Subjektverständnis, dass Menschen autonome Individuen seien, die sozialen Verhältnissen und sozialen Praktiken vorgängig seien - das Recht regele ja gerade, wie der freie Wille des einen mit dem freien Willen aller anderen zusammenstimmen könne, damit ein friedliches Miteinander aller überhaupt möglich werde. Das autonome Subjekt wäre damit eine Ermöglichungsbedingung sozialer Praktiken. Dieses facettenreiche Subjektverständnis wird im Recht stets vorausgesetzt - ob bei den verfassungsrechtlich geschützten Grundrechten der einzelnen Individuen, beim zivilrechtlich fundamentalen Gedanken der Privatautonomie oder bei den für das Schuldstrafrecht zentralen Annahmen von Willensfreiheit und Schuld.

${ }^{1}$ Taylor, Sources of the Self: The Making of Modern Identity, 1989; Siedentop, Inventing the Individual: The Origins of Western Liberalism, 2014; Reckwitz, Subjekt, 2008; ders., Die Gesellschaft der Singulariäten: Zum Strukturwandel der Moderne, 2017. 
- Aber sind nicht all dies Selbstverständlichkeiten, die man in Lehrbüchern für Studienanfängerinnen nachlesen kann?

Aus Sicht der Praxeologie handelt es sich bei dem Subjektverständnis des Rechts nicht um eine Selbstverständlichkeit, sondern um ein historisches und kulturspezifisches soziales Konstrukt. Die Praxeologie, oder Praxistheorie, ist ein junger Ansatz im interdisziplinären Feld aus Sozialwissenschaften, Kulturtheorie, Ethnologie und Philosophie, der auf Arbeiten von Ludwig Wittgenstein und Martin Heidegger aufbaut, für den Denkerinnen wie Michel Foucault, Pierre Bourdieu und Judith Butler zentral sind, und der zuletzt durch Eike von Savigny, Karin Knorr-Cetina und Theodore Schatzki als ,practice turn“ bekannt gemacht und vorangetrieben wurde. ${ }^{2}$ Die praxeologische Perspektive besteht darin, in der Analyse von Phänomenen weder autonome Subjekte noch durch abstrakte Regeln charakterisierbare Gesellschaften an erste Stelle zu setzen. Ausgangspunkt sind stattdessen von menschlichen Körpern in materiellen Umwelten habituell stets aufs Neue vollzogene soziale Praktiken. ${ }^{3}$ Menschen seien demnach nicht autonome Subjekte, sondern Körper, die sozialisiert würden und dadurch soziale Normen vor allem als implizites Wissen-Wie beherrschten. Ihr Handeln sei ein habituelles Verhalten, das innerhalb sozialer Praktiken in steter Interaktion mit lokalen, materiellen Umwelten stattfinde. Insofern werden Subjekte in der Praxeologie „,dezentriert": Zentral für eine Analyse menschlichen Verhaltens sind vielmehr Praktiken, Körper, implizites Wissen-Wie und materielle und soziale Umwelten. Dennoch werden Körper auch aus praxeologischer Sicht durch die Teilnahme an Praktiken zu bestimmten Subjekten - wenn auch „Subjekt" hier nicht die rechtliche und moralische Vorstellung eines autonomen Subjekts bezeichnet, sondern einen Körper, der sein Leben durch ein sozial konstituiertes Selbstbild leiten lässt. Indem nämlich ein Körper stets an den Vollzügen einer bestimmten Praxis teilnehme, werde er „subjektiviert“, d. h. den Normen der Praxis unterworfen (,subicere“) und gerade dadurch zu einem Subjekt gemacht. Lebt ein Körper etwa in einer Gesellschaft, in der ein binäres GenderVerständnis von höchster sozialer Relevanz ist, und wird der Körper stets als eindeutig zu einem der Gender gehörig behandelt, wird er sich fortan selbst entweder als Mann oder Frau begreifen, im sozialen Umgang mit anderen so handeln, wie man gelernt hat, als Mann bzw. Frau zu handeln, und eben dadurch dazu beitragen, das Gender-Verständnis wieder zu reproduzieren. ${ }^{4}$ Die Praxis, in der menschliche Körper in eine binäre Gender-Matrix eingeordnet werden, ist damit dem einzelnen Körper, der sich als Mann oder Frau begreift, vorgeordnet. Dabei werden Körper erst im ständigen Vollzug, in der ständigen Performanz von Praktiken zu bestimmten Subjekten. Aus praxeologischer Sicht gibt es damit keine autonomen Subjekte, die an Praktiken teilnehmen könnten und dass einige Körper bestimmte lebensleitende Selbstbilder haben, ist vielmehr erst das Resultat dessen, dass die Körper an entsprechenden sozialen Praktiken teilgenommen haben. Das „Subjekt“ ist dann bestenfalls ein Produkt sozialer Praktiken.

Nun liegt es sehr nahe, aus einer solchen praxeologischen Perspektive das rechtliche und moralische Subjektverständnis zu problematisieren. Immerhin handelt es sich bei der Praxeologie um ein interdisziplinäres, sich mehr und mehr in empirischen Untersuchungen bewährendes Forschungsprogramm, das sich explizit, differenziert und empi-

\footnotetext{
${ }^{2}$ Von Savigny/Knorr-Cetina/Schatzki, The Practice Turn in Contemporary Theory, 2001. Vgl. Reckwitz, Die Transformation der Kulturtheorien, 2003.

${ }^{3}$ Schatzki, Social Practices: A Wittgensteinian Approach to Human Activity and the Social, 1996.

${ }^{4}$ Butler, Das Unbehagen der Geschlechter, 1991.
} 
risch informiert mit dem Thema des Subjekts auseinandergesetzt hat. Die Praxeologie hat auch bereits die klassische Subjektphilosophie etwa Kants und anderer kritisiert. ${ }^{5}$ Allerdings haben sich die Praxeologinnen bisher kaum dem Rechtssubjekt zugewandt ${ }^{6}$ und die Rechtwissenschaftlerinnen umgekehrt kaum der Praxeologie. Hier besteht mithin eine wichtige Lücke in der Forschung.

Zur Schließung dieser Lücke ist die Beantwortung einer Fülle von Fragen notwendig: Ist das Subjektverständnis des Rechts und der Moral falsch, weil Menschen in Wirklichkeit nichts anderes sind als sozialisierte Körper? Kann man Menschen noch gerechtfertigter Weise einen Schuldvorwurf machen, wenn sie keine autonomen Subjekte sind? Wenn aber eine praxeologische Betrachtung zu solch dramatischen Konsequenzen führt, zeigt dies dann nicht, dass mit der Praxeologie selbst etwas nicht stimmt? Oder ist das Subjektverständnis des Rechts vielleicht selbst lediglich das Produkt von Subjektivierungsprozessen, die durch rechtliche Praktiken entstehen? Könnte man dann durch Änderungen der rechtlichen Praktiken ein besseres Subjektverständnis des Rechts hervorbringen? Oder muss nicht auch die Praxeologie voraussetzen, dass Menschen eigentlich autonome Subjekte sind - etwa, wenn begründete Kritik an sozialen Praktiken möglich sein soll? Oder geht vielleicht andersherum die Praxeologie in ihrer Kritik an klassischen Subjektverständnissen noch nicht weit genug?

Derartige Fragen waren Thema auf der interdisziplinären Tagung „Recht und Moral als soziale Praktiken?" des Jungen Forums Rechtsphilosophie, die im September 2017 in Regensburg stattgefunden hat. Eine sich mit dem Thema des autonomen Subjekts beschäftigende Auswahl der Beiträge haben wir in diesem Themenschwerpunkt versammelt. Ihr Spektrum reicht von der Verteidigung des autonomen Subjekts bis hin zu seiner vollständigen Abschaffung.

Martin Weichold,

Universität Regensburg,

E-Mail: martin.weichold@psk.uni-regensburg.de

\author{
Jan-Christoph Marschelke, \\ Universität Regensburg, \\ E-Mail: jan.marschelke@sprachlit.uni-regensburg.de \\ Peter Wiersbinski, \\ Universität Regensburg, \\ E-Mail: peter.wiersbinski@psk.uni-regensburg.de
}

Falk Hamann,

Universität Regensburg,

E-Mail: falk.hamann@psk.uni-regensburg.de

\footnotetext{
${ }^{5}$ Vgl. für Darstellungen Reckwitz, Das hybride Subjekt: Eine Theorie der Subjektkulturen von der bürgerlichen Moderne zur Postmoderne, 2006, und ders., Subjekt, 2008.

${ }^{6}$ Freilich könnte man Foucault, Überwachen und Strafen, 1977, hier einordnen.
} 